\title{
A 2-Bit Pancharatnam-Berry Coding Metasurface For Ultra-Wideband And Polarization-Insensitive RCS Reduction
}

Baoqin Lin ( $\sim$ aflbq@sina.com )

Xijing University https://orcid.org/0000-0002-4167-1834

Wenzhun Huang

Xijing University

Yong-sheng Yang

Xijing University

Lin-tao LV

Xijing University

Jianxin Guo

Xijing University

Yanwen Wang

Xijing University

\section{Research Article}

Keywords: Metasurface, Pancharatnam-Berry phase, RCS Reduction

Posted Date: August 9th, 2021

DOI: https://doi.org/10.21203/rs.3.rs-591172/v1

License: () (1) This work is licensed under a Creative Commons Attribution 4.0 International License.

Read Full License

Version of Record: A version of this preprint was published at Plasmonics on January 11th, 2022. See the published version at https://doi.org/10.1007/s11468-021-01586-4. 


\title{
A 2-bit Pancharatnam-Berry Coding Metasurface for Ultra-wideband and Polarization-insensitive RCS Reduction
}

\author{
Bao-qin Lin ${ }^{1}$, Wen-zhun Huang ${ }^{1}$, Yong-sheng Yang ${ }^{1}$, Lin-tao Lv ${ }^{1}$, Jian-xin Guo ${ }^{1}$, Yan-wen Wang ${ }^{1}$
}

\begin{abstract}
Because Pancharatnam-Berry (PB) geometrical phase can only be generated in the co-polarized reflection coefficient under circular polarized (CP) incidence for a reflective metasurface, designing a reflective $\mathrm{PB}$ coding metasurface must be based on an appropriate polarization conversion metasurface $(\mathrm{PCM})$, which can realize $\mathrm{CP}$ maintaining reflection. In this work, to design a reflective 2dit PB coding metasurface for radar cross section (RCS) reduction, an ultra-wideband PCM is proposed at first, the simulated results show that the PCM can realize ultrawideband CP-maintaining reflection from 8.6 to $35.9 \mathrm{GHz}$; moreover, $\mathrm{PB}$ phase will be generated in its co-polarized reflection coefficient by rotating its unit structure. Thus based on the PCM, an ultra-wideband reflective 2-dit PB coding metasurface is constructed successfully. The simulation and experiment results show that the coding metasurface has excellent performance in RCS reduction under arbitrary polarized incidences, compared with a pure metallic plate with the same size, its RCS can be reduced more than $10 \mathrm{~dB}$ in the frequency band $8.2-35.2 \mathrm{GHz}$ with a relative bandwidth of $124.4 \%$.
\end{abstract}

Keywords Metasurface; Pancharatnam-Berry phase; RCS Reduction.

\section{Introduction}

Metasurfaces are two-dimensional planar metamaterials with ultrathin thickness, which usually consist of a planar array of sub-wavelength resonant unit cell structures. Through reasonable design of the resonant unit cell structures, almost all basic properties of electromagnetic (EM) waves can be tailored by various metasurfaces. Due to the favorable characteristics of metasurfaces, varieties of metasurfaces have been proposed to reduce the radar cross section (RCS) of stealth targets.

To reduce the RCS of stealth targets by using metasurfaces, there are two commonly used methods: one is absorption, and the other is phase cancellation. For the first method, many metamaterial absorbers have been proposed, which can transform electromagnetic energy into heat and realize effective absorption [1-7], however, metamaterial absorbers are usually difficult to achieve wide-band absorption because they can only operate in the vicinity of resonance frequency. For the second method, a chessboardlike metasurface composed of artificial magnetic conductors (AMC) and perfect electronic conductor (PEC) was firstly proposed by Panquay et al. in 2007 [8], whose RCS was reduced because of the cancellation effects arising from the $180^{\circ}$ reflection phase difference between PEC and AMC, but

\footnotetext{
${ }^{1}$ School of Information Engineering, Xijing University, Xi'an 710123 , China. Email: aflbq@sina.com
}

the RCS reduction bandwidth was narrow due to the limited bandwidth of the AMC. Later, to expand the RCS reduction bandwidth, a number of chessboard-like metasurfaces composed of two different types of AMCs have been reported [9-15]. However, due to the regular arrangement, four strong redirected scattering beams always can't be ruled out for any chessboard-like metasurfaces. In 2014, a concept of coding metasurface was firstly proposed by Cui et al.,[16] which consist of several different types of coding elements with much different reflection phases, it can work as a reflective phased array antenna, and the array pattern can be represented by a coding sequence, so its far-field scattering pattern can be controlled by the predesigned coding sequence, and according to a proper coding sequence, the anticipated RCS reduction can be realized due to phase cancellation. In recent years, to realize wideband RCS reduction, a number of different coding metasurfaces have been proposed [17-27].

In this work, an ultra-wideband reflective 2-bit Pancharatnam-Berry (PB) coding metasurface is designed for RCS reduction, the design process is presented in detail, in which an ultra-wideband polarization conversion metasurface (PCM) is proposed at first. Based on the PCM, four suitable coding elements are constructed by using $\mathrm{PB}$ phase, thus a 2-bit PB coding metasurface is proposed according to an optimized coding sequence. The simulated and experimental results show that the coding metasurface can realize ultra-wideband and polarization-insensitive RCS reduction under arbitrary polarized incidences.

\section{Design and simulation}

\section{1) Coding element design}

To design a coding metasurface, several suitable coding elements must be proposed at first. In this work, to construct several suitable coding elements for a reflective PB coding metasurface, an ultra-wideband PCM is proposed at first, which consists of a patterned metallic layer printed on a grounded dielectric substrate and covered by a dielectric layer. The PCM is a two-dimensional periodic structure, one of its unit cells is shown in Fig. 1, it is shown that the metallic patch in the unit cell is a circular structure, which is very suitable for rotation to generate PB phase. In addition, the geometrical parameters of the unit cell are shown in Fig. 1. After suitable selection, these geometrical parameters have been chosen as follows: $P=6.00 \mathrm{~mm}, r=2.60 \mathrm{~mm}$, $t=0.20 \mathrm{~mm}, w=0.90 \mathrm{~mm}, g=0.35 \mathrm{~mm}, h_{1}=h_{2}=2.40 \mathrm{~mm}$; the metallic patch, together with the grounded plane, is modeled as a $0.017 \mathrm{~mm}$ copper film with an electric conductivity $\sigma=5.8 \times 10^{7} \mathrm{~S} / \mathrm{m}$; in addition, the two dielectric layers are both chosen as a Teflon with a relative permittivity of 2.0. 


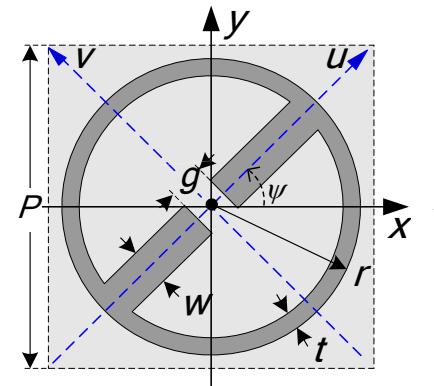

(a)

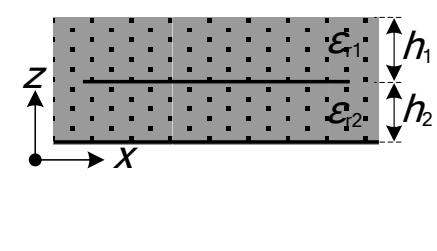

(b)
Fig. 1 Unit cell of the proposed PCM: (a) Top view; (b) Side view.

The unit cell of the PCM is an orthogonal anisotropic structure with a pair of mutually perpendicular symmetric axes $u$ and $v$. The angle between the positive $u$-axis and positive $x$-axis is defined as the rotation angle $\psi$ of the unit cell structure, now it has been rotated by $\psi=45^{\circ}$, as shown in Fig. 1(a). For a reflective metasurface composed of such an orthogonal anisotropic unit cell structure, according to Ref. [28], the reflection coefficients under circular polarized (CP) incidences can be expressed as follows:

$$
\begin{aligned}
& r_{++}=\frac{1}{2}\left(r_{u u}-r_{v v}\right) e^{+\mathrm{j} 2 \psi} \\
& r_{--}=\frac{1}{2}\left(r_{u u}-r_{v v}\right) e^{-\mathrm{j} 2 \psi} \\
& r_{-+}=\frac{1}{2}\left(r_{u u}+r_{v v}\right) \\
& r_{--}=\frac{1}{2}\left(r_{u u}+r_{v v}\right)
\end{aligned}
$$

wherein $r_{u u}$ and $r_{v v}$ denote the co-polarized reflection coefficient under $u$ - and $v$-polarized incidences respectively, whose magnitudes will both be close to 1.0 when the dielectric loss in the metasurface structure is little because no cross-polarized reflection component will exists under $u$ - and $v$-polarized incidences due to the symmetry of the metasurface structure. Thus when the little dielectric loss is neglected, the following equation can be established:

$$
r_{v v}=r_{u u} e^{-\mathrm{j} \Delta \varphi_{u v}}
$$

wherein $\Delta \varphi_{u v}$ denotes the phase difference between $r_{u u}$ and $r_{v v}$. Based on Eq. (1) and (2), the magnitudes of $r_{++}, r_{--}$, $r_{-+}$and $r_{+-}$can be expressed as:

$$
\begin{aligned}
& \left|r_{++}\right|=\frac{1}{2}\left|r_{u u}\right|\left|1-e^{-\mathrm{j} \Delta \varphi_{u v}}\right|\left|e^{+\mathrm{j} 2 \psi}\right|=\sqrt{\left(1-\cos \Delta \varphi_{u v}\right) / 2} \\
& \left|r_{--}\right|=\frac{1}{2}\left|r_{u u}\right|\left|1-e^{-\mathrm{j} \Delta \varphi_{u v}}\right|\left|e^{-\mathrm{j} 2 \psi}\right|=\sqrt{\left(1-\cos \Delta \varphi_{u v}\right) / 2}, \\
& \left|r_{-+}\right|=\left|r_{+-}\right|=\frac{1}{2}\left|r_{u u}\right|\left|1+e^{-\mathrm{j} \Delta \varphi_{u v}}\right|=\sqrt{\left(1+\cos \Delta \varphi_{u v}\right) / 2}
\end{aligned}
$$

Eq. (1) illustrates that when the unit cell structure of the metasurface is rotated by an angle $\psi, \pm 2 \psi$ PB phase will be generated in $r_{++}$and $r_{--}$, but the phase of $r_{+-}$and $r_{-+}$ will not be changed, which implies that to design a reflective PB coding metasurface, it must be based on an appropriate PCM, which can make the magnitudes of its $r_{++}$and $r_{--}$ close to 1.0. In addition, Eq. (3) indicates that the magnitudes of $r_{++}$and $r_{--}$can be determined by the phase difference $\Delta \varphi_{u v}$ between $r_{u u}$ and $r_{v v}$. No matter how many degrees the unit cell structure of the metasurface is rotated, the magnitudes of its $r_{++}$and $r_{--}$will be close to 1.0 when $\Delta \varphi_{u v}$ is close to $\pm 180^{\circ}$.

Now to analyse the performance of the proposed PCM, according to the above analysis, we have simulated it under $u$ - and $v$-polarized incidences at first. The simulated results, shown in Fig. 2(a), indicate that the phase difference $\Delta \varphi_{u v}$ between $r_{u u}$ and $r_{v v}$ is close to $180^{\circ}$ in the ultra-wide frequency range from 8 to $36 \mathrm{GHz}$. In addition, the magnitudes of $r_{++}$and $r_{-+}$calculated by using Eq. (3 ) are shown in Fig. 2(b), it is shown that the anticipated CPmaintaining reflection can be realized in the frequency band from 8.6 to $35.9 \mathrm{GHz}$, wherein the magnitude of $r_{++}$can be kept close to 1.0 , and that of $r_{-+}$can be kept less than 0.3 .

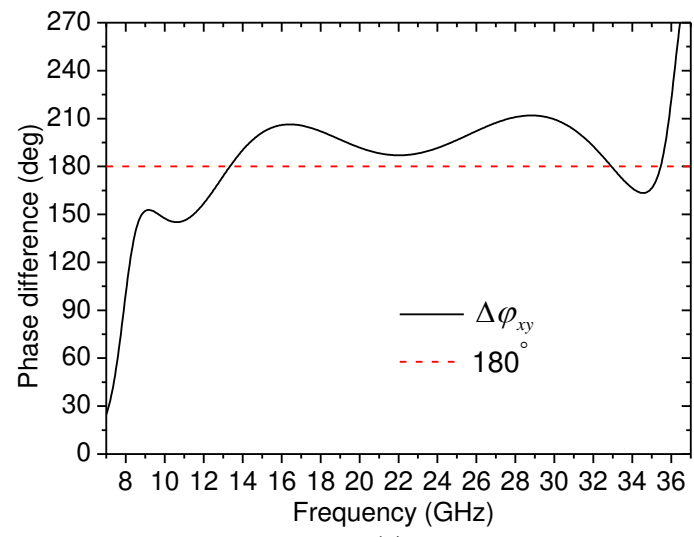

(a)

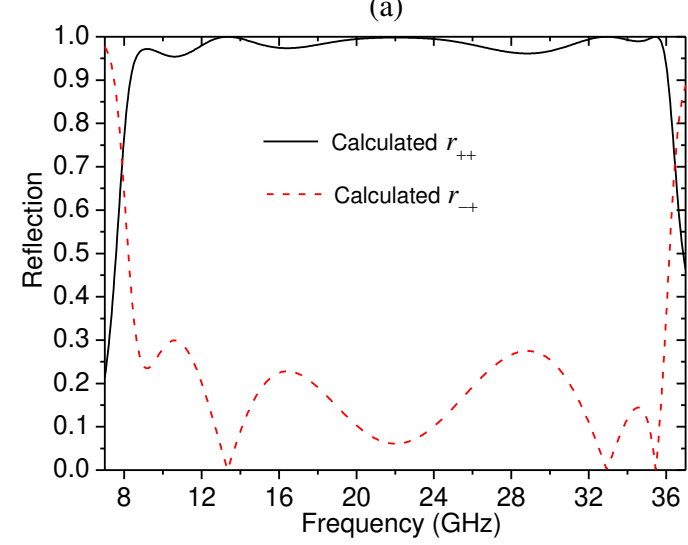

(b)

Fig. 2 Simulated and calculated results of the PCM: (a) the phase difference between $r_{u u}$ and $r_{v v}$, (b) the magnitudes of $r_{++}$and $r_{-+}$.

In order to verify the correctness of the above theoretical prediction, the PCM has been repeatedly simulated under right-handed $\mathrm{CP}$ (RCP) and left-handed $\mathrm{CP}$ (LCP) incidences when the rotation angle $\psi$ of its unit cell structure was gradually increased from $0^{\circ}$ to $157.5^{\circ}$ with a $22.5^{\circ}$ step width. The simulated results, the magnitude of $r_{++}$, is shown in Fig. 3(a), but the magnitude of $r_{--}$is not shown for it is almost the same as that of $r_{++}$; in addition, the phases of $r_{++}$ and $r_{--}$are shown in Fig. 3(b) and (c), respectively. In Fig. 3(a), it is shown that no matter how much angle the unit cell 
structure is rotated, the simulated magnitude of $r_{++}$is almost always the same as the above calculated one, which verifies the above theoretical prediction. Moreover, Fig. 3(b) and (c) show that the phase of $r_{++}$keeps increasing gradually but the phase of $r_{--}$keeps decreasing gradually along with the increase of the rotation angle $\psi$ in the ultra-wide frequency range from 8 to $36 \mathrm{GHz}$, and they are both altered by almost $45.0^{\circ}$ at each time.

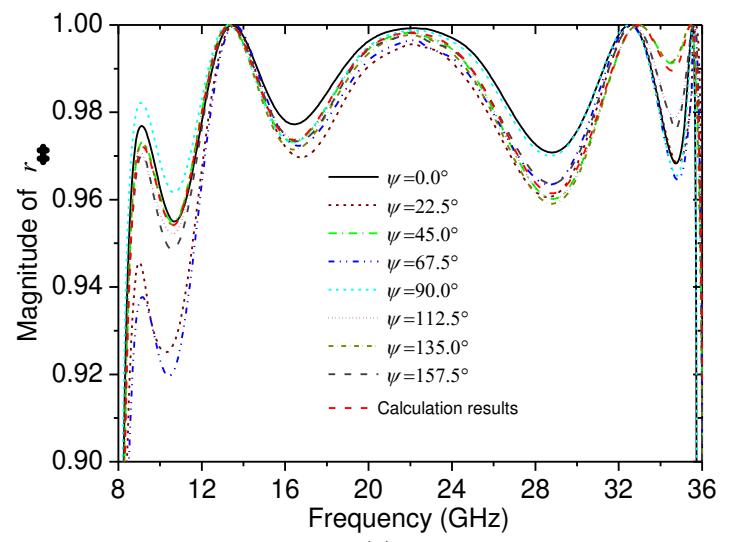

(a)

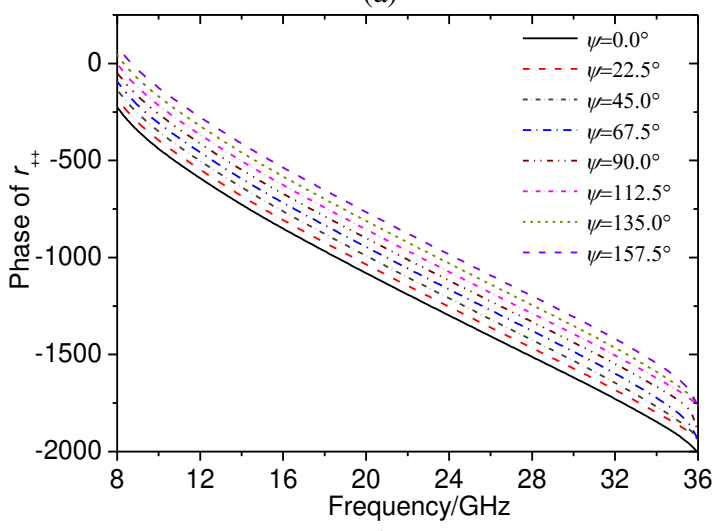

(b)

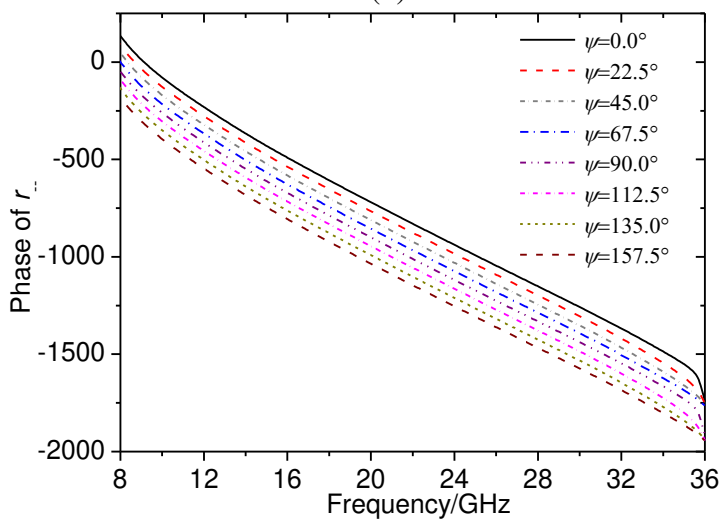

(c)

Fig. 3 Simulated results of the PCM with different rotating angle $\psi$ : (a) the magnitude of $r_{++}$, (b) the phase of $r_{++}$, (c) the phase of $r_{-+}$.

The above theoretical analysis and simulations show that the proposed PCM can realize ultra-wideband CPmaintaining reflection in the frequency range from 8.6 to $35.9 \mathrm{GHz}$; moreover, when the rotation angle $\varphi$ is increased by $\Delta \varphi$, the $\mathrm{PB}$ phases generated in $r_{++}$and $r_{--}$will be $+2 \Delta \varphi$ and $-2 \Delta \varphi$, respectively. Thus, the proposed PCM can be used as coding elements, and when the rotation angle $\psi$ of its unit cell structure is set as $0^{\circ}, 45^{\circ}, 90^{\circ}$ and $135^{\circ}$, its relative reflection phases under RCP incidence will be $0^{\circ}, 90^{\circ}$, $180^{\circ}$ and $270^{\circ}$ respectively, whose codes can be defined as " $00 "$, " 01 ", " $10 "$ and " 11 ".

\section{2) Coding metasurface design}

After the four coding elements are obtained, we construct a 2-bit PB coding metasurface based on a random coding sequence, which consists of $6 \times 6$ coding elements, and each element is composed of $5 \times 5$ identical unit cells. When the 2 bit coding metasurface is under RCP or LCP incidence, the reflection phase of each coding element can be known according to the random coding sequence. We assume that the reflection phase of the $(m, n)$ th element is $\phi(m, n)$, thus according to the theory of phased array antenna, the far-field scattering pattern of the coding metasurface can be expressed as:

$$
\mathrm{F}(\theta, \varphi)=f_{e}(\theta, \varphi) \cdot A F_{6 \times 6}(\theta, \varphi),
$$

wherein $\theta$ and $\varphi$ are the elevation and azimuth angle of the scattering direction respectively, $f_{e}(\theta, \varphi)$ represents the scattering pattern of the coding element, which can be written as $\cos (\theta)$, and $A F_{6 \times 6}(\theta, \varphi)$ represents the array factor, when the plane wave is incident vertically, the array factor can be expressed as:

$$
\begin{gathered}
A F_{6 \times 6}=\sum_{m=1}^{6} \sum_{n=1}^{6} \exp \{-i\{\phi(m, n) \\
\left.\left.+k_{0} D \sin \theta[(m-1 / 2) \cos \varphi+(n-1 / 2) \sin \varphi]\right\}\right\}
\end{gathered},
$$

in which $D$ denotes the distance between two adjacent coding elements. For the 2-bit PB coding metasurface, the reflection amplitudes in various coding elements are basically the same, but the reflection phases are different, the reflection phase different between " 00 " and " 10 ", together that between " 01 " and " 11 ", is close to $180^{\circ}$ under both RCP and LCP incidences, so the best RCS reduction can be realized due to phase cancellation after optimizing the random coding sequence of the coding metasurface under arbitrary polarized incidences.

To optimize the coding sequence of the coding metasurface, genetic algorithm (GA) has been used. GA is a widely used optimization algorithm, which uses the theory of biological evolution for reference, simulates the problem to be solved as a biological evolution process, produces the next generation solution through copying, crossover, mutation and so on, and gradually eliminate the solution with poor fitness and increase the solution with good fitness, thus the desired optimal solution can be obtained after $\mathrm{N}$ generation evolution. In the application, our objective is to realize the best RCS reduction and let the scattering in all directions be small. For the far-field scattering pattern of the coding metasurface is expressed as Eq. (4), the fitness function can be written as follow:

$$
\text { fitness }=1 / \max (F(\theta, \varphi)) \text {. }
$$

According to the fitness function, it is well known that the larger the fitness, the smaller the peak of $A F_{6 \times 6}(\theta, \varphi)$ will be. In this way, when the population size is chosen as 300 , the 
crossover probability is chosen as 0.8 , and the mutation probability is set as 0.05 , the fitness function converges to a maximum value after 120 generations of iterations, an optimized coding sequence is obtained, which is shown in Fig. 4(b). In addition, based on the optimized coding sequence, the final coding metasurface is constructed, as shown in Fig. 4(b).

\begin{tabular}{|l|l|l|l|l|l|}
\hline 01 & 01 & 01 & 01 & 10 & 10 \\
\hline 01 & 01 & 10 & 10 & 10 & 10 \\
\hline 10 & 01 & 11 & 11 & 00 & 11 \\
\hline 10 & 01 & 00 & 00 & 00 & 11 \\
\hline 11 & 11 & 01 & 10 & 00 & 00 \\
\hline 11 & 11 & 00 & 11 & 00 & 00 \\
\hline
\end{tabular}

(a)

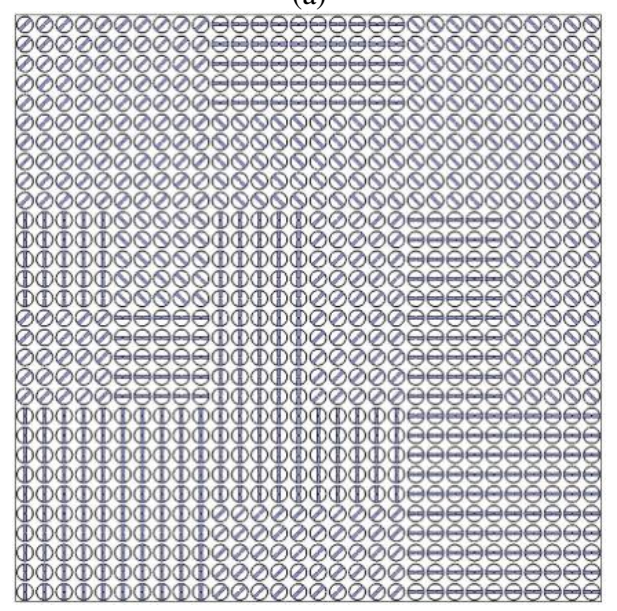

(b)

Fig. 4 The obtained optimized coding sequence (a), and the schematic diagrams of the final 2-bit PB coding metasurface (b).

To verify the performance of our design, the final 2-bit PB coding metasurface, together with a pure metallic plate with the same size, has been simulated under different incidences using CST Microwave Studio. When the excitation is chosen as RCP, LCP and LP normal incident waves successively, the obtained simulated results, shown in Fig. 5, indicate that the monostatic RCS of the coding surface is almost the same under different polarization incidences, compared with the pure metallic plate, more than $10 \mathrm{~dB}$ RCS reduction is achieved in the ultra-wide band 8.2-

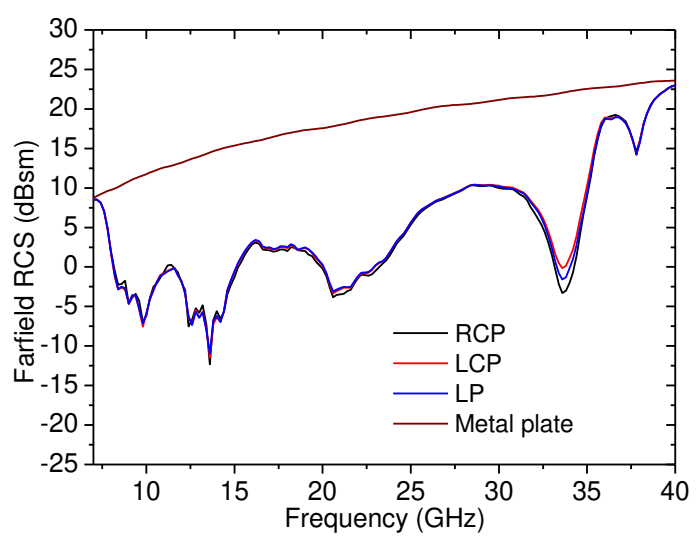

(a)

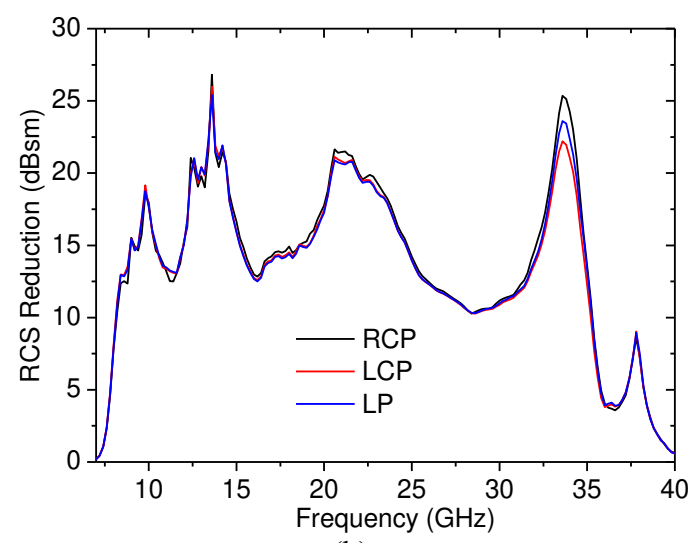

(b)

Fig. 5 Simulated results of the coding metasurface and the metallic plate: (a) the monostatic RCS, (b) the RCS reduction.

$35.2 \mathrm{GHz}$ with a relative bandwidth of $124.4 \%$, and the maximum reduction can reach $26.8 \mathrm{~dB}$.

In addition, for further analysis of the far-field scattering characteristics of the 2-dit PB coding metasurface, the bistatic scattering curves of the coding metasurface and the metallic plate in the YOZ plane at $10.0 \mathrm{GHz}, 22.0 \mathrm{GHz}$, and $34.0 \mathrm{GHz}$ are obtained through far-field simulation. In the simulation, the excitations at the three frequency points are set as RCP, LCP and LP normal incident waves, respectively. The simulated results, shown in Fig. 6, indicate that compared with the strong specular reflection of the metallic plate, the energy of the main lobe and most side lobes of the coding metasurface is obviously suppressed although the energy of some side lobes is enhanced, which show that the bistatic RCS of the coding metasurface is reduced in most directions under different polarization incidences.

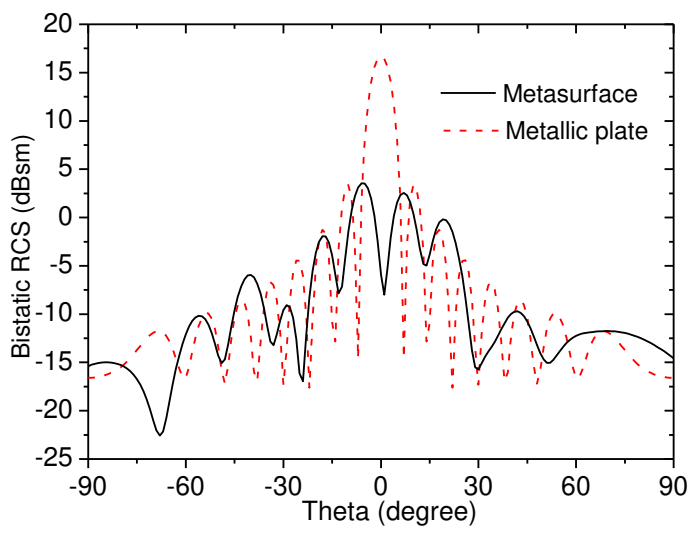

(a)

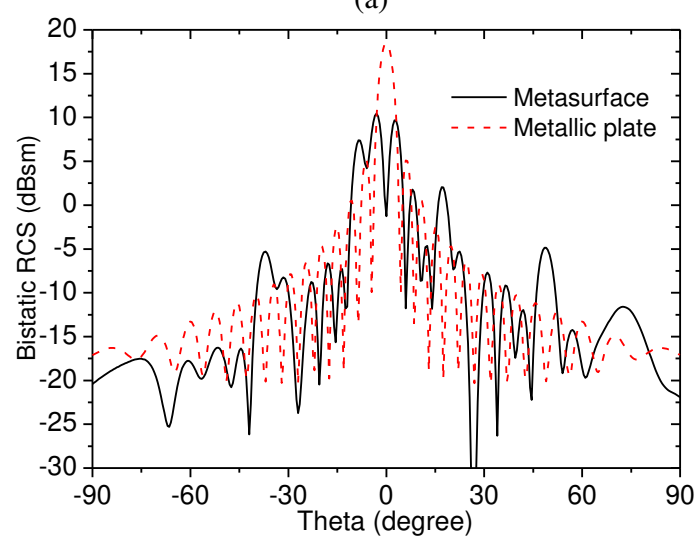

(b) 


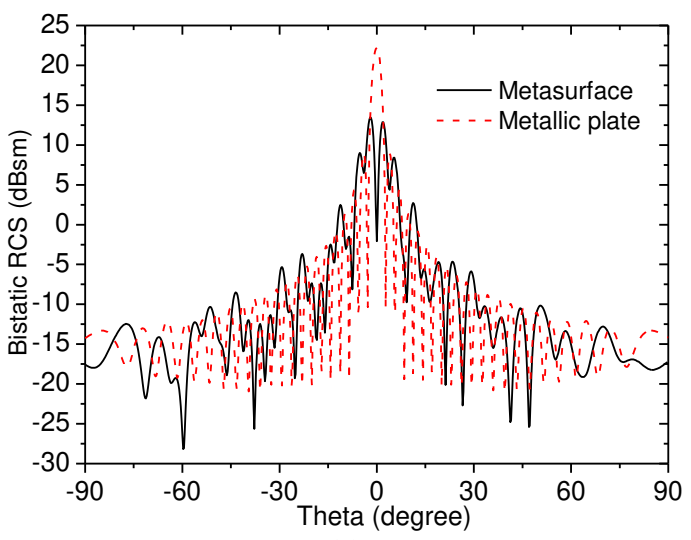

(c)

Fig. 6 Bistatic RCS of coding metasurface and metallic plate in YOZ plane: (a) $10.0 \mathrm{GHz}$ under RCP incidence; (b) $22.0 \mathrm{GHz}$ under LCP incidence; (c) $34.0 \mathrm{GHz}$ under LP incidence.

Moreover, to analyze the angle insensitivity, the 2-dit PB coding metasurface and the pure metallic plate have been simulated under different oblique incidences. The simulated results, shown in Fig. 7(a), indicate that the monostatic RCS reduction of the coding metasurface is still larger than $10 \mathrm{~dB}$ in the most parts of the frequency band $8.2-37.8 \mathrm{GHz}$ when the incident angle is increased to $45^{\circ}$ under RCP incidence; in addition, those in Fig. 7(b) indicate that the monostatic RCS reduction are almost the same under different polarized incidences with incident angle of $45^{\circ}$, which show that the coding metasurface has good angular stability under arbitrary polarized incidences.

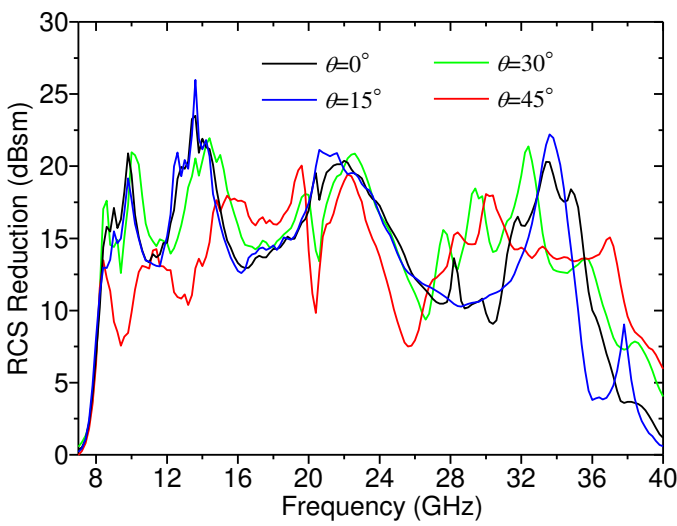

(a)

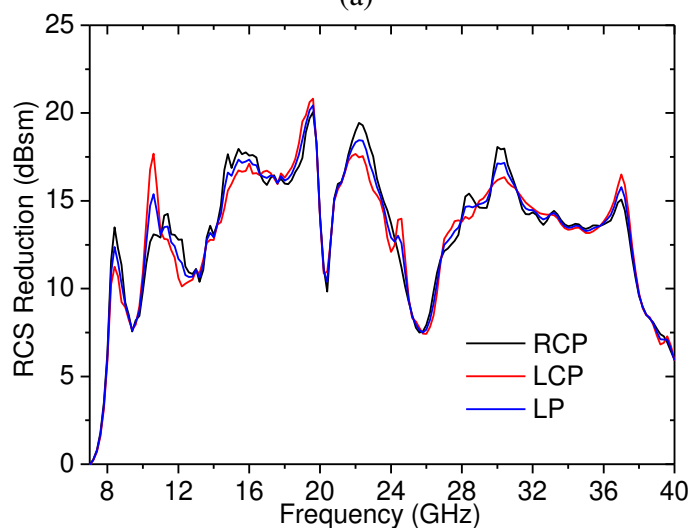

(b)

Fig. 7 RCS reduction of the coding metasurface under different oblique incidences: (a) under RCP incidence with different incident angles, (b) under different polarization incidences with incident angle of $45^{\circ}$.

\section{Experimental results}

Finally, to realize an experimental validation for our design, the prototype of the proposed 2-bit PB coding metasurface is fabricated. In the manufacturing process, a grounded dielectric substrate is manufactured by $\mathrm{PCB}$ technology, which is shown in Fig. 8(a), and then the whole structure is obtained by hot pressing it with a top dielectric layer. The fabricated prototype has been measured using free-space measurement techniques, it has been measured under RCP and LP normal incidences and oblique incidences with incident angle of $45^{\circ}$. In addition, a metallic plate with the same size has also been measured in the same way as a reference. The measured results, shown in Fig. 8(b) and (c), indicate that when the coding metasurface is under normal or oblique incidence, the measured results under RCP and LP incidences are almost the same, which are both in reasonable agreement with the simulated results.
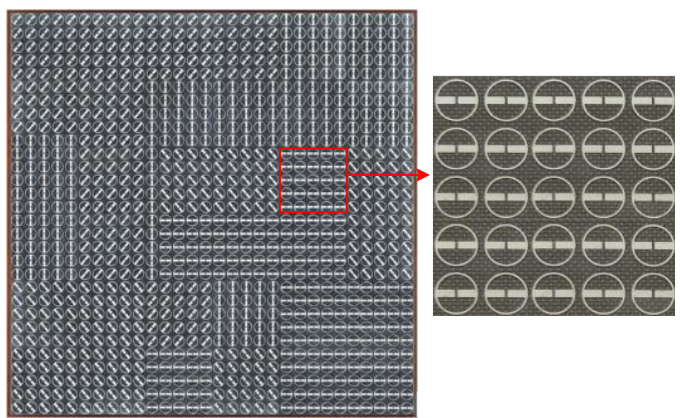

(a)

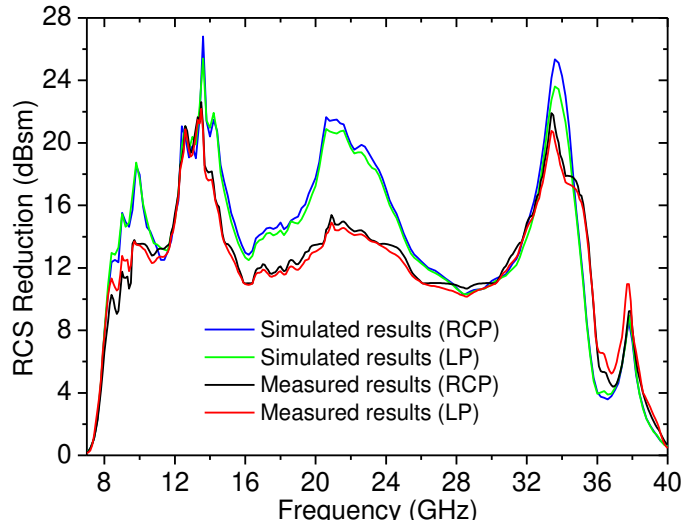

(b)

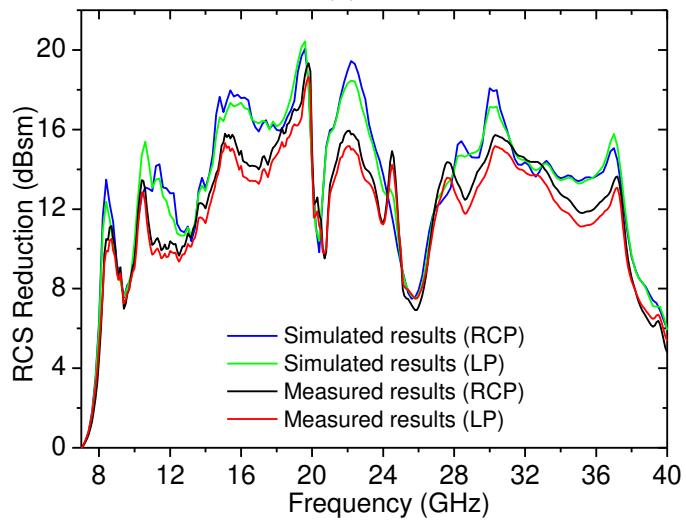

(c)

Fig. 8 The photograph of the fabricated prototype (a) and the measured results: (b) under normal incidence, (c) under oblique incidence with incident angle of $45^{\circ}$. 


\section{Conclusion}

In this paper, an ultra-wideband reflective 2-bit $\mathrm{PB}$ coding metasurface based on a PCM was proposed for RCS reduction. Numerical simulations showed the coding metasurface can realize more than $10 \mathrm{~dB}$ RCS reduction under arbitrary polarized incidences in the frequency band from 8.2 to $35.2 \mathrm{GHz}$ compared with a pure metallic plate with the same size. Finally, one experiment was carried out, there was a reasonable agreement between the simulated and experimental results. Compared with the previous designs, the 2-bit PB coding metasurface can achieve much wider band RCS reduction under arbitrary polarized incidences, so it is of great application values in radar stealth technology application.

\section{Declarations}

Ethical Approval: This is a basic study. The National Natural Science Foundation Research Committee of China has confirmed that no ethical approval is required.

Consent to Participate: Informed consent was obtained from all individual participants included in the study.

Consent to Publish: Each participant has consented to the submission to the journal.

Authors Contributions: Conceptualization: Bao-qin Lin, Wen-zhun Huang; Methodology: Yong-sheng Yang, Jianxin Guo; Formal analysis and investigation: Bao-qin Lin, Yan-wen Wang; Writing-original draft preparation: Bao-qin Lin, Yan-wen Wang; Writing-review and editing: Wen-zhun Huang, Lin-tao Lv; Funding acquisition: Bao-qin Lin, Jianxin Guo, Yan-wen Wang; Resources: Wen-zhun Huang, Yan-wen Wang; Supervision: Wen-zhun Huang, Lin-tao Lv. Funding: This work was supported by National Natural Science Foundation of China (Grant No. 62072378), Natural Science Foundation of Shaanxi Province, China (2019JM077), and Xi'an Science and Technology Plan Project, China(GXYD20.4).

Competing Interests: The authors declare that they have no competing interests.

Availability of data and materials: The datasets used and/or analysed during the current study are available from the corresponding author on reasonable request.

\section{Acknowledgments}

This work was supported by the National Natural Science Foundation of China (Grant No. 62072378), Natural Science Foundation of Shaanxi Province, China (2019JM-077), Xi'an Science and Technology Plan Project, China(GXYD20.4).

\section{References}

1. Ishii Y, Masaki T, et al. (2017) Experimental consideration of RCS reduction using thin metasurface. IEICE Communications Express 1:1-6.
2. Wang M, Weng B, Zhao J, et al. (2017) Dendriticmetasurface-based flexible broadband microwave absorbers. Applied Physics A 123:434

3. Wang Y, Zhu DY, Cui ZJ, (2020) Properties and Sensing Performance of All-Dielectric Metasurface $\mathrm{THz}$ Absorbers, IEEE Transactions on Terahertz Science and Technology 10:599-605.

4. H. Yang, X. Cao, J. Gao, et al. (2013) Low RCS Metamaterial Absorber and Extending Bandwidth Based on Electromagnetic Resonances, Progress In Electromagnetics Research M 33:31-44.

5. Ishii Y, Masaki T, Michishita N, et al. (2016) RCS Reduction Characteristics of Thin Wave Absorbers Composed of Flat and Curved Metasurfaces, ISAP 2016: 192-193.

6. Li Y, Wang J, Zhang J, et al. (2014) Ultra-wide-band microwave composite absorbers based on phase gradient metasurfaces, Progress in Electromagnetics Research M 40: 9-18.

7. Li Z, Stan L, et al. (2019) Broadband infrared binarypattern metasurface absorbers with micro-genetic algorithm optimization, Optics Letters 44:114-117.

8. Paquay M, Iriarte JC, Ederra I, et al. (2007) Thin AMC Structure for Radar Cross-Section Reduction. IEEE Trans. Antennas Propag. 55:3630-3638.

9. Ma E, Alvarez Y, Las HF, (2011) A novel approach for RCS reduction using a combination of artificial magnetic conductors, Progress In Electromagnetics Research 107:147-159.

10. Galarregui JCI, Pereda AT, De Falcon JLM, (2013) Broadband Radar Cross-Section Reduction Using AMC Technology, IEEE Trans. Antennas Propag. 61: 61366143.

11. Modi A Y, Balanis C A, Birtcher C R, et al. (2017) Novel Design of Ultrabroadband Radar Cross Section Reduction Surfaces Using Artificial Magnetic Conductors, IEEE Transactions on Antennas and Propagation 65: 5406-5417.

12. Zaker R, Sadeghzadeh A, (2018) Study and Design of a Double-layer Ultra-thin AMC Structure for Wideband Radar Cross Section Reduction, IET Microwaves Antennas \& Propagation 12: 1601.

13. Libi M, Vadakkekalathil A H, Chandroth K, (2018) Wideband radar cross section reduction using artificial magnetic conductor checkerboard surface, Progress In Electromagnetics Research M 69:171-183.

14. Ameri E, Esmaeli S H, Sedighy S H, (2018) Ultra Wide Band Radar Cross Section Reduction Using Multilayer Artificial Magnetic Conductor Metasurface, Journal of Physics D Applied Physics 51: 285304.

15. Sang D, Chen Q, Ding L, et al. (2019) Design of Checkerboard AMC Structure for Wideband RCS Reduction, IEEE Transactions on Antennas and Propagation 67: 2604-2612.

16. Cui T J, Qi M Q, Wan X, Zhao J, and Cheng Q, (2014) Coding metamaterials, digital metamaterials and programmable metamaterials, Light, Sci. Appl. 3: e218-e218.

17. Yan X, Liang L, Yang J, et al. (2015) Broadband, wideangle, low-scattering terahertz wave by a flexible 2-bit coding metasurface, Optics Express 23: 29128.

18. Su J, Lu Y, Li Z, et al. (2016) A Wideband and Polarization-Independent Metasurface Based on Phase 
Optimization for Monostatic and Bistatic Radar Cross Section Reduction, International journal of antennas and propagation, 2016:7595921.1-7595921.9.

19. Yuan F, Wang GM, Xu H X, et al. (2017) Broadband RCS reduction based on spiral-coded metasurface, IEEE Antennas Wireless Propag. Lett. 16: 3188-3191.

20. Sun H, Gu C, Chen X, et al. (2017) Broadband and broad-angle polarization-independent metasurface for radar cross section reduction, Scientific Reports 7: 407821.

21. Feng M, Li Y, Zheng Q, Zhang J, et al. (2018) Twodimensional coding phase gradient metasurface for RCS reduction, J. Phys. D, Appl. Phys. 51: 375103.

22. Khan T A, Li J, Chen J, et al. (2019) Design of a Low Scattering Metasurface for Stealth Applications, Materials 12:3031.

23. Saifullah Y, Waqas A B, Yang G, et al. (2019) 4-Bit Optimized Coding Metasurface for Wideband RCS Reduction, IEEE Access 7: 122378-122386.

24. Hao H, Du S, and Zhang T, (2019) Small-Size Broadband Coding Metasurface for RCS Reduction Based on Particle Swarm Optimization Algorithm, Progress In Electromagnetics Research M 81: 97-105.

25. Han X, Xu H, Chang Y, et al. (2020) Multiple Diffuse Coding Metasurface of Independent Polarization for RCS Reduction, IEEE Access 8: 162313-162321.

26. Yang J, Li Y, Cheng Y, et al. (2020) Design and analysis of 2-bit matrix-type coding metasurface for stealth application, Journal of Applied Physics 127: 235304.

27. Fu C, Han L, Liu C, et al. (2020) Reflection-type 1-bit coding metasurface for radar cross section reduction combined diffusion and reflection, Journal of Physics D Applied Physics 53: 445107.

28. Lin B, Lv L, Guo J, et al. (2020) Ultra-wideband Anomalous Reflection Realized by a Gradient Metasurface, IET Microwaves Antennas \& Propagation 14: 1424-1430. 